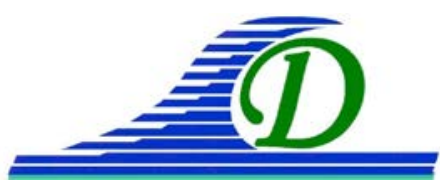

XIII ${ }^{\text {èmes }}$ Journées Nationales Génie Côtier - Génie Civil Dunkerque, 2-4 juillet 2014

DOI:10.5150/jngcgc.2014.110 C Editions Paralia CFL

disponible en ligne - http://www.paralia.fr - available online

\title{
Caractérisation des sédiments de dragage du barrage de Bouhanifia pour une réutilisation
}

\section{Fatiha KAZI AOUAL-BENSLAFA ${ }^{1}$, Mohammed AMEUR ${ }^{1}$, Belkacem MEKERTA ${ }^{1}$, Abdelaziz SEMCHA ${ }^{1}$}

\author{
1. Laboratoire "LabMat", ENP Oran; BP 1523 Oran El Ménaouer, \\ 31000 Oran, Algérie. \\ fkaziaoual@yahoo.fr ; mohammed.ameur@gmail.com; mekertab@yahoo.fr ; \\ asemcha@yahoo.fr
}

\section{Résumé :}

La préservation des ressources en eau dans les régions semi arides passe par la recherche de solutions préventives contre l'envasement des retenues de barrages telle que la maîtrise de la dynamique sédimentaire du bassin versant ou curative tel que l'entretien des fonds de la retenue et des organes du barrage. Dans ce travail nous nous sommes intéressés à la seconde solution qui est le dés-envasement du barrage de Bouhanifia par le dragage et le stockage des sédiments dragués. Après la présentation du barrage de Bouhanifia et de son bassin versant, nous présentons les opérations de dragage du barrage de Bouhanifia qui doivent concerner plus de six millions de $\mathrm{m}^{3}$ de sédiments et leur stockage aux abords de l'ouvrage. Une première caractérisation des sédiments de dragage a été réalisée dans le but de définir des voies de valorisation.

Mots-clés : Barrage de Bouhanifia, Sédiments fluviaux, Dragage, Caractérisation, Voies de valorisation.

\section{Introduction}

Dans le contexte actuel, l'Algérie est confrontée à une double problématique environnementale. D’abord, celle de la fabrication des ciments CEMI, en raison de leur teneur en clinker élevée (95\% en masse) engendre un effet néfaste sur l'environnement. En effet, la production d'une tonne de clinker génère le rejet d'une tonne de $\mathrm{CO}_{2}$ dans l'atmosphère. Pour palier à cette problématique environnementale, une des solutions pouvant être envisagée est l'emploi d'addition minérale en substitution partielle du ciment (GARTNER, 2004 ; CASSAGNABERE, 2007). La deuxième problématique est celle de la préservation de la ressource en eau. L'Algérie compte actuellement 70 barrages en exploitation pour une capacité de stockage estimée à 7,1 milliards de $\mathrm{m}^{3}$. Mais celle ci a tendance à diminuer à cause du phénomène d'envasement qui touche l'ensemble des barrages algériens. Des levés bathymétriques ont été réalisés en 2005 et 2006 par l'ANBT (2008) sur 57 grands barrages de cinq bassins hydrographiques et le volume total de vase est estimé à 1,1 milliards de $\mathrm{m}^{3}$, soit un comblement de $15 \%$ de la capacité totale. Une étude réalisée par MORSLI et al. (2004) donne une carte de 
sensibilité à l'envasement des barrages dans laquelle est représentée la zone à très fort taux d'envasement des 18 barrages les plus envasés en Algérie parmi lesquels on compte le barrage de Fergoug (MEKERTA et al., 2012) et celui de Bouhanifia. Le comblement de ces deux barrages est de $100 \%$ et $60 \%$ respectivement (REMINI \& HALLOUCHE, 2007). Des études ponctuelles ont été menées en Algérie dans le cadre de la valorisation des sédiments de dragage dans les matériaux de construction tels que la brique et la pouzzolane artificielle comme ajout au ciment pour la fabrication du ciment composé ainsi qu'en technique routière. Pour la fabrication de la brique, les sédiments de onze barrages algériens les plus envasés ont été utilisés, les résultats obtenus montrent que la vase présente les mêmes caractéristiques que celle de l'argile jaune utilisé par l'ensemble des briqueteries d'Algérie (LABIOD et al., 2004; REMINI, 2006; SEMCHA, 2006). Pour la fabrication d'une pouzzolane artificielle, l'étude a concerné les sédiments du barrage de Fergoug (BENKADDOUR et al., 2009), le pourcentage de substitution de $10 \%$ de ciment par la vase calcinée dans les mortiers a permis d'obtenir une résistance mécanique similaire à celle du mortier de référence. Alors que le pourcentage de $20 \%$ a permis d'améliorer la résistance des mortiers soumis aux attaques chimiques de l'acide $\mathrm{HCl}$. En technique routière, le comportement mécanique des sédiments de dragage du barrage Fergoug pour leur valorisation comme matériau de remblaiement a été étudié (MEKERTA et al., 2009 ; BOURABAH, 2012).

Dans ce travail, après la présentation du site du barrage de Bouhanifia et de l'opération de dragage, nous présentons une première caractérisation des sédiments de dragage. Les sédiments sont stockés dans des bassins en attente d'une éventuelle réutilisation. La filière de valorisation que nous visons est celle des matériaux de construction en Génie Civil comme additions minérales dans le ciment CPA ou comme filler, les deux réutilisations pouvant être exploitées industriellement et favoriseraient une gestion durable du barrage de Bouhanifia.

\section{Description du site du barrage de Bouhanifia}

Le barrage de Bouhanifia est situé au nord-ouest de l'Algérie à environ $100 \mathrm{~km}$ au sud d'Oran et à $25 \mathrm{~km}$ de Mascara (figure 1). C'est un barrage en enrochement arrimé de $460 \mathrm{~m}$ de longueur totale en crête, il s'élève à $56 \mathrm{~m}$ au-dessus du thalweg, sa largeur au sommet est de $5 \mathrm{~m}$, elle atteint $125 \mathrm{~m}$ à la base. La retenue du barrage de Bouhanifia est alimentée principalement par l'oued El hammam qui prend sa source au niveau des Trois Rivières, point de confluence des oueds Melrir, Hounet (formé par la confluence des oueds Sefioum et Berbour) et l'oued Sahouet (formé par la confluence des oueds Taria et Saïda) et grossi par l'oued Fekane (figure 2). Le bassin versant de l'oued El Hammam en amont du barrage de Bouhanifia couvre une superficie d'environ $7700 \mathrm{~km}^{2}$. Il est sous l'influence du climat méditerranéen dont la température oscille entre $8^{\circ} \mathrm{C}$ et $10^{\circ} \mathrm{C}$ en hiver à $+30^{\circ} \mathrm{C}$ en été, avec une moyenne des précipitations de $350 \mathrm{~mm}$ par an. Les opérations de dragage ont démarré en juin 2013 mais au préalable 


\section{XIII ${ }^{\text {èmes }}$ Journées Nationales Génie Côtier - Génie Civil \\ Dunkerque, 2-4 juillet 2014}

des bassins de rejet et de décantation ont fait l'objet d'une étude et leur implantation se trouve à quelques kilomètres de la retenue du barrage de Bouhanifia. Plus de six millions de $\mathrm{m}^{3}$ doivent être dragués sur une durée de deux années. Les moyens utilisés sont assez faibles, composés de deux dragues hydrauliques et à ce jour l'extraction n'excède pas le million de $\mathrm{m}^{3}$. La figure 3 indique la situation géographique des bassins de rejet, la zone comprend à ce jour quatre grands bassins qui sont aptes à recevoir toute la quantité de sédiments dragués. La figure 4 représente une photo du matériel de dragage mis en place.

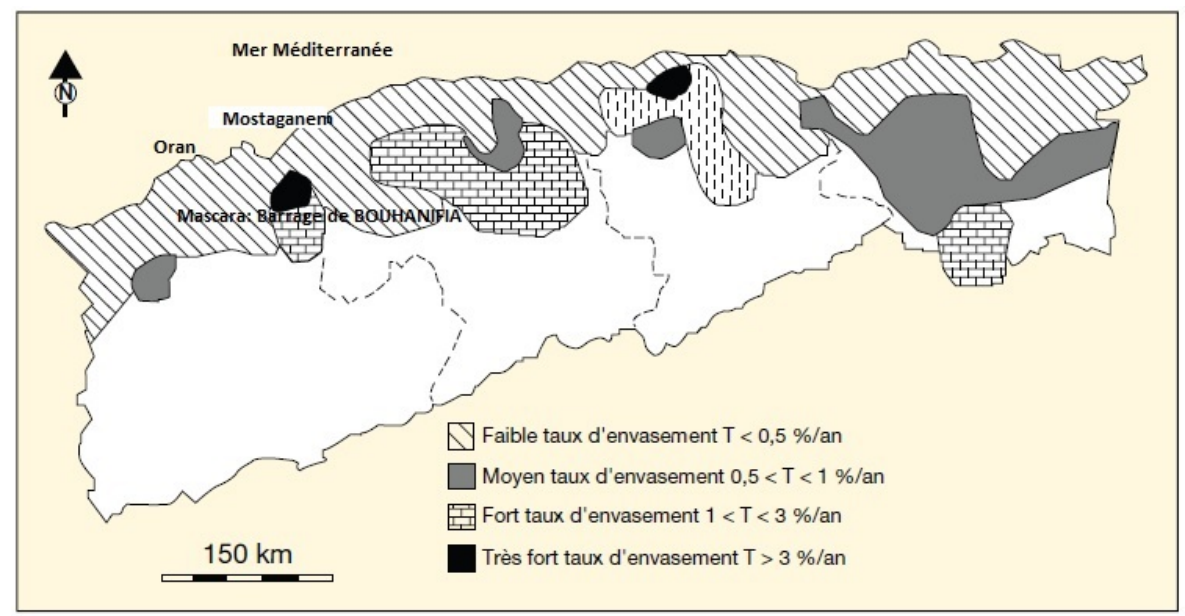

Figure 1. Carte de sensibilité à l'envasement des barrages en Algérie (MORSLI et al., 2004).

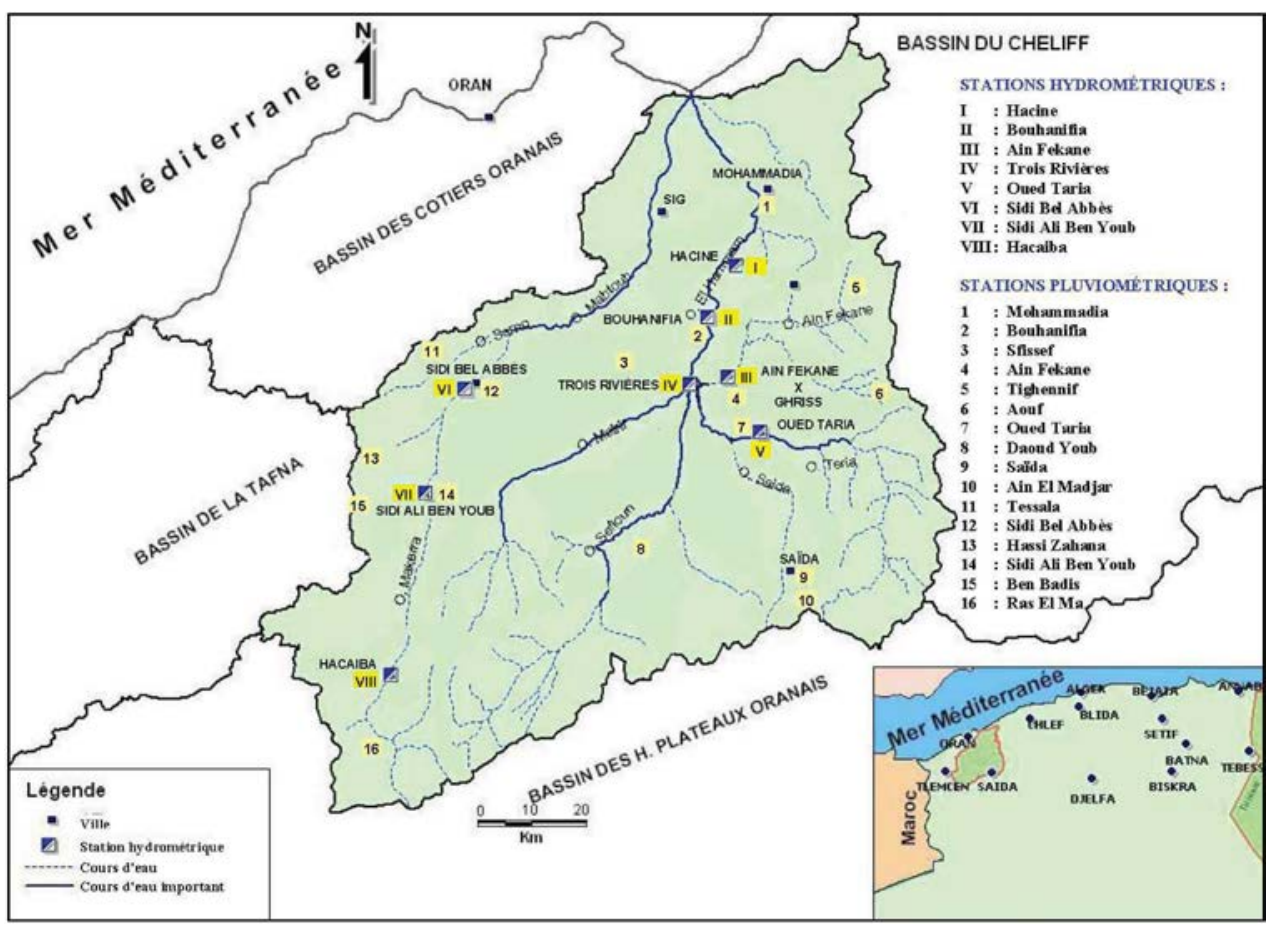

Figure 2.Réseau hydrographique du bassin versant de la Macta (MEDDI et al., 2009). 


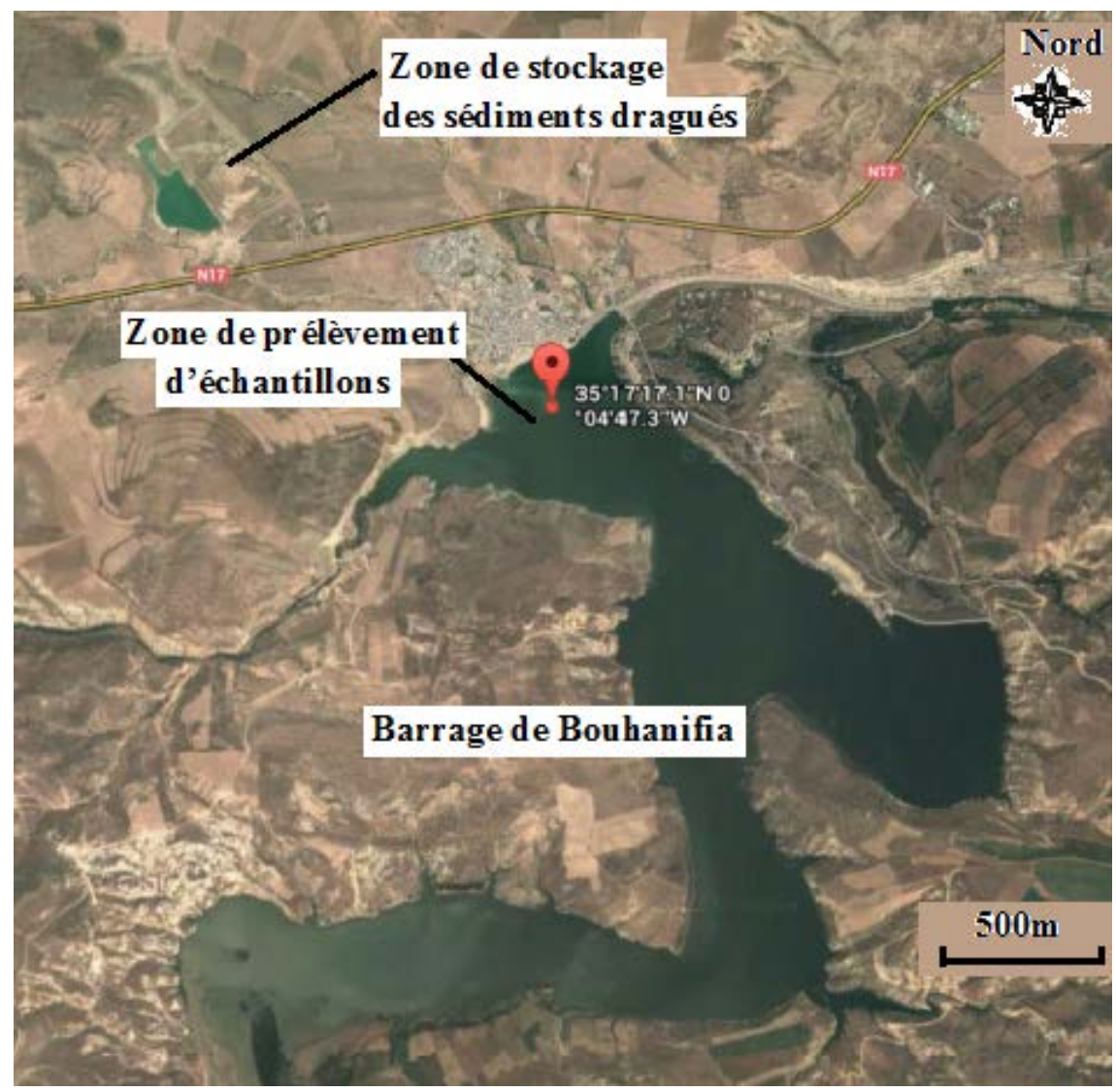

Figure 3. Situation géographique de l'emplacement des dragues dans la cuvette du barrage de Bouhanifia et des bassins de rejet des sédiments dragués.
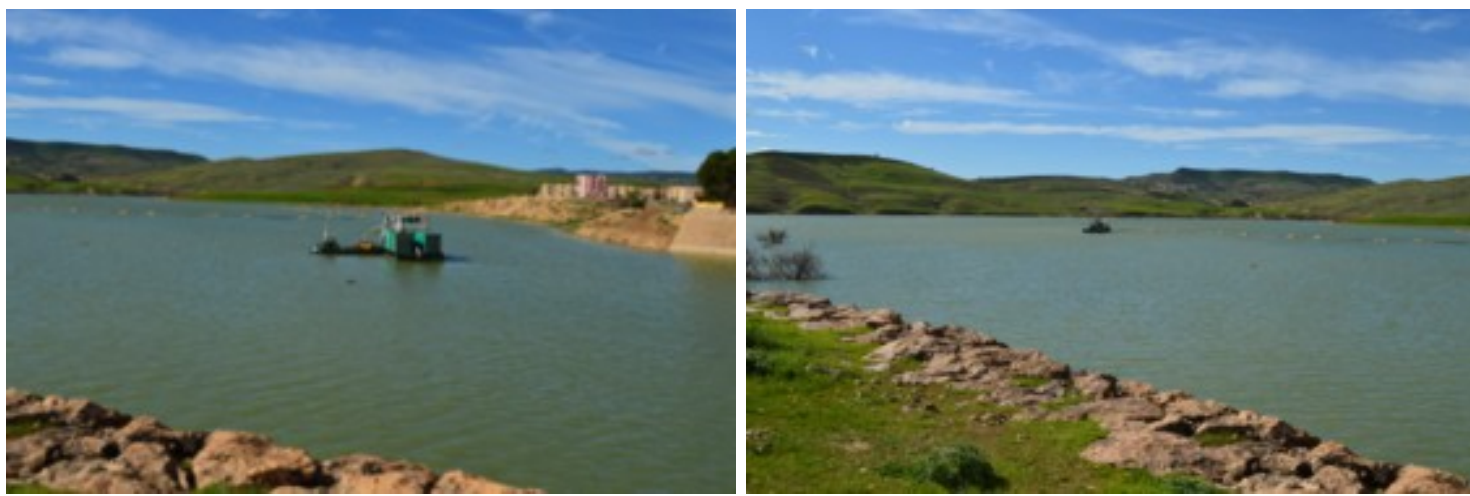

Figure 4. Photos du matériel de dragage dans la retenue du Barrage de Bouhanifia (Photos Equipe Mekerta).

3. Les méthodes utilisées pour une réutilisation des sédiments dragués dans les mortiers

Les échantillons de sédiments dragués (SD) ont été collectés dans la retenue du barrage de Bouhanifia ; la situation géographique et la localisation de la zone de prélèvement des échantillons est située autour du point donné par les coordonnées suivantes: 


\section{XIII $I^{\text {èmes }}$ Journées Nationales Génie Côtier - Génie Civil \\ Dunkerque, 2-4 juillet 2014}

$35^{\circ} 17^{\prime} 17.1^{\prime \prime} \mathrm{N}$ et $0^{\circ} 04^{\prime} 47.3^{\prime \prime} \mathrm{W}$. Ils ont été transportés dans des containers hermétiquement scellés et stockés au laboratoire ensuite une première caractérisation a été réalisée.

La caractérisation des éléments chimiques majeurs des SD a été déterminée conformément à la norme Européenne EN1744 (1998); les valeurs moyennes sont reportées au tableau1.

La granulométrie est une donnée importante, elle a été réalisée suivant la norme Européenne EN P 94-056 (1996), et par sédimentométrie suivant la norme Européenne EN P 94-057 (1992).

Les SD ont été tamisés et broyés à $80 \mu \mathrm{m}$ et ont été introduits dans les mortiers en substitution du ciment

Tableau1. Caractéristiques physiques et chimiques des SD de la retenue du Barrage de Bouhanifia.

\begin{tabular}{llll}
\hline Caractéristiques & Symboles & Unités & Teneurs \\
\hline Silice & $\mathrm{SiO}_{2}$ & $\%$ & 86.85 \\
Chaux & $\mathrm{CaO}$ & $\%$ & 6.53 \\
Magnésie & $\mathrm{Mg} 0$ & $\%$ & - \\
Alumine & $\mathrm{Al}_{2} \mathrm{O}_{3}$ & $\%$ & 0.48 \\
Oxyde de Fer & $\mathrm{Fe}_{2} \mathrm{O}_{3}$ & $\%$ & 2.26 \\
Sulfates & $\mathrm{SO}_{3}$ & $\%$ & 0.90 \\
Chlorures & $\mathrm{CL}$ & $\%$ & 0.05 \\
Perte au feu & $\mathrm{P.F}$ & $\%$ & 13.20 \\
Carbonates & $\mathrm{CaCO}_{3}$ & $\%$ & 11.36 \\
L'anhydride Carbonique & $\mathrm{CO}_{2}$ & $\%$ & 5 \\
Eau de combinaison & $\mathrm{H}_{2} \mathrm{O}$ & $\%$ & 6.81 \\
Matière Organique & $\mathrm{MO}$ & $\%$ & 3.09 \\
Masse volumique & $\rho_{\mathrm{s}}$ & $\mathrm{kg} / \mathrm{m}^{3}$ & 2580 \\
\hline
\end{tabular}

Dans cette première partie de l'étude, l'objectif est de mettre en évidence l'influence de la substitution partielle du ciment par les SD sur le comportement des mortiers à l'état. Les mortiers ont été confectionnés en substituant le ciment par différentes fractions de sédiments: $0 \%$ (mortier de référence MR), 10\%, 15\% (mortiers MS10, MS15). Les essais de l'ouvrabilité des mortiers sont réalisés selon la norme américaine ASTM C 1437 (2001). Un moule en forme de cône est utilisé pour donner la forme initiale de l'éprouvette. Le moule est retiré et on réalise 25 secousses en 15 secondes. Le mortier s'étale et le diamètre est mesuré et comparé au diamètre initial. La fluidité $F$ du mortier est donnée par la relation : $F(\%)=\left(\left(D_{25}-D_{0}\right) / D_{0} \times 100\right)$, où $D_{25}$ est le diamètre du mortier après les 25 coups et $D_{0}$ est le diamètre initial du mortier. Pour la plupart des mortiers la 
fluidité requise est de $110 \%$. Cet essai est répété jusqu'à ce que l'obtention de la fluidité requise soit atteinte. La quantité d'eau est alors enregistrée.

\section{Résultats et discussion}

Les courbes granulométriques sont illustrées par la Figure5, elles montrent que les SD sont des sables fins limoneux. L'analyse chimique a révélé que les SD ont un taux élevé en silice, ce taux est similaire à celui de la vase du barrage de Fergoug. Des essais complémentaires seront effectués pour surveiller les effets de cette silice sur le comportement mécanique des mortiers à l'état durci et leur durabilité. Les SD sont faiblement organiques avec une valeur de MO égale à 3.09\%. Les chlorures sont présents en faible quantité mais la présence de sulfates est anormalement élevée. Ceci est dû probablement à une utilisation intensive de ces derniers par les agriculteurs de la région.

Le comportement des mortiers: les compositions des mortiers et des résultats de l’ouvrabilité sont donnés dans le tableau 2.

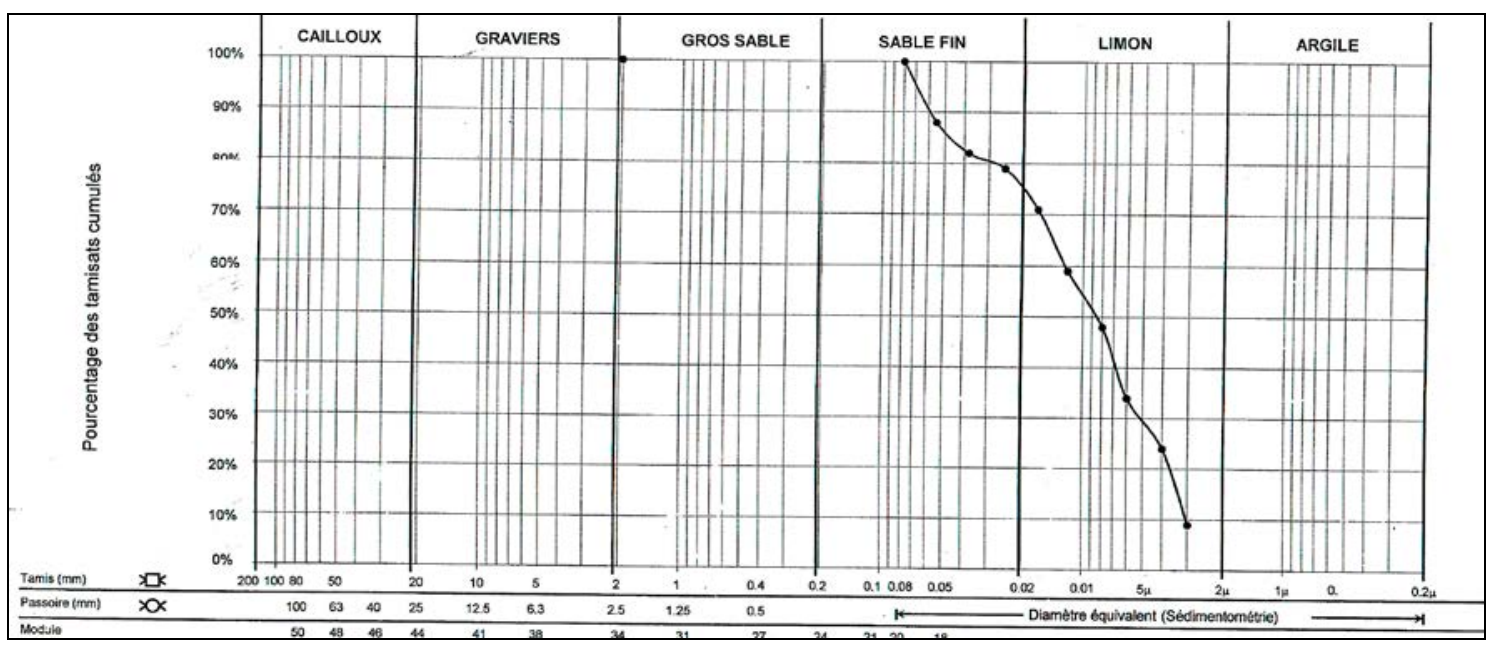

Figure 5. Courbes granulométriques des $S D$.

Tableau 2. Compositions et ouvrabilité des différents mortiers.

\begin{tabular}{llll}
\hline \multirow{2}{*}{$\begin{array}{l}\text { Matériaux } \\
\text { et caractéristiques }\end{array}$} & \multicolumn{3}{l}{ Désignation des mortiers } \\
\cline { 2 - 4 } & $R M$ & $M S 10$ & $M S 15$ \\
\hline Ciment $\left(\mathrm{kg} / \mathrm{m}^{3}\right)$ & 450 & 405 & 382,5 \\
Sand $\left(\mathrm{kg} / \mathrm{m}^{3}\right)$ & 1350 & 1350 & 1350 \\
$S D\left(\mathrm{~kg} / \mathrm{m}^{3}\right)$ & 0 & 45 & 67,5 \\
Ratio E/C (\%) & 0,50 & 0,50 & 0,50 \\
Ouvrabilité (\%) & $110 \%$ & $88 \%$ & $75 \%$ \\
Ratio E/C (\%) avec l'ouvrabilité (110\%) & 0,50 & 0,56 & 0,58 \\
\hline
\end{tabular}




\section{XIII $I^{\text {èmes }}$ Journées Nationales Génie Côtier - Génie Civil \\ Dunkerque, 2-4 juillet 2014}

L'ouvrabilité des mortiers et bétons dépend la granulométrie des granulats, de la quantité d'eau du mélange et le pourcentage de SD ajoutés (LIMEIRA et al., 2011). Dans cette étude, on a observé une importante réduction de la fluidité des mortiers avec l'augmentation du volume de SD. La distribution de la taille des grains des SD augmente la compacité du mélange granulaire (ciment + Sable + SD) et une partie de l'eau libre nécessaire à l'hydratation du ciment est absorbée par les SD. Donc il est nécessaire d'augmenter le ratio $\mathrm{E} / \mathrm{C}$ pour obtenir une fluidité constante à $110 \%$. Des résultats similaires ont été trouvés par LIMEIRA en 2011.

\section{Conclusions}

L'effet bénéfique du dragage sera comptabilisé en volume récupéré de la capacité de stockage dans la retenue du barrage de Bouhanifia.

Des améliorations ont été constatées pour la préservation de l'environnement avec la mise en place de bassins de stockage temporaire des sédiments dragués, le projet dirigé par l'ANBT a pris en compte ce côté environnemental.

La caractérisation physique des SD de la retenue du barrage de Bouhanifia a révélé qu'ils ont une granulométrie dont les principaux constituants sont des sables fins et des limons. La caractérisation chimique des SD a révélé un taux de chlorures acceptable. Les sulfates sont anormalement élevés, des essais sur mortier devront prouver qu'ils ne présentent aucun danger pour le mortier. La demande en eau est supérieure dans les mortiers avec ajout de SD pour obtenir l'ouvrabilité requise.

Toutefois, l'expérimentation devra se poursuivre pour l'élaboration de la fiche d'identité des SD (LEVACHER et al., 2011).

\section{Références bibliographiques}

ANBT -Agence Nationale des Barrages et Transferts- (2008). Réconfortement du barrage de Bouhanifia, document interne. Agence Nationale des Barrages et Transfert, Alger, $20 \mathrm{p}$.

ASTM C1437-01 (2001). Standard Test Method for flow of hydraulic Cement Mortar. BENKADDOUR M., KAZI-AOUAL F., SEMCHA A. (2009). Durabilité des mortiers à base de pouzzolane naturelle et de pouzzolane artificielle. Revue Nature et Technologie. $n^{\circ}$ 01/Juin 2009, pp 63-73.

BOURABAH M.A. (2012). Comportement mécanique des sols fins, application à la valorisation des sédiments de barrages en technique routière. Thèse de doctorat en sciences, Université de Tlemcen, Algérie, 237 p.

CASSAGNABERE F., ESCADEILLAS G., MOURET M., BROILLIARD P. (2007). Low $\mathrm{CO}_{2}$ energy binder for precast industry. $12^{\text {th }}$ International Congress on the Chemistry of Cement, July 2007, Montreal.

GARTNER E. (2004). Industrially interesting approaches to "low-CO2" cement. Cement and Concrete Research, Vol. 34, pp 1489-1498. http://dx.doi.org/10.1016/j.cemconres.2004.01.021 
Thème 8 - Gestion et valorisation des sédiments marins

EN, P.94-056 (1996). Soils: recognition and testing - Particle size analysis of soils, dry sieving method.

EN, P.94-057 (1992). Soils: recognition and testing - Soil textural analysis - Method by sedimentation.

EN1744 (1998). Tests for chemical properties of aggregates - Part 1: Chemical analysis

LABIOD Z., REMINI B., BELAREDJ M. (2004). Traitement de la vase du barrage de Bouhanifia en vue de sa valorisation. Larhyss Journal, n 03, Juin 2004, pp 7-12.

LEVACHER D., SANCHEZ M., DUAN Z., LIANG Y. (2011). Valorisation en unité pilote de sédiments méditerranéens: Etude des caractéristiques géotechniques et de la perméabilité. Revue Paralia, Vol. 4, pp 4.1-4.20. http://dx.doi.org/10.5150/revue-paralia.2011.004 LIMEIRA J., ETXEBERRIA M., AGULLÓ L., MOLINA D. (2011). Mechanical and durability properties of concrete made with dredged marine sand. Construction and Building Materials, Vol. 25, pp 4165-4174. http://dx.doi.org/10.1016/j.conbuildmat.2011.04.053 MEDDI M., TALIA A., MARTIN C. (2009) Évolution récente des conditions climatiques et des écoulements sur le bassin versant de la Macta (Nord-Ouest de l'Algérie). Revue géographie physique et environnement, Vol. 3, pp 61-84.

MEKERTA B., SEMCHA A., BENAISSA A., KAZI-AOUEL-BENSLAFA F. (2009). Comportement mécanique des sédiments de dragage pour leur valorisation en technique routière. SBEIDCO - 1st International Conference on Sustainable Built Environment Infrastructures in Developing Countries ENSET Oran (Algeria), pp 12-14. MEKERTA B., KAZI-AOUAL F., SEMCHA A., BENAISSA A., RAHMANI F. (2012). Estimation de l'érosion spécifique saisonnière et valorisation des sédiments de dragage: Cas particulier du barrage de Fergoug (Algérie). Revue COST ENSET Oran, $\mathrm{n}^{\circ} 10$ janvier 2012, pp 19-26.

MORSLI B., MAZOUR M., MEDEDJEL N., HAMOUDI A., ROOSE E. (2004). Influence de l'utilisation des terres sur les risques de ruissellement et d'érosion sur les versants semi-arides du nord-ouest de l'Algérie. Sécheresse 2004, Vol. 15, pp 96-104.

REMINI B. (2006). Valorisation de la vase des barrages. Quelques exemples algériens. Larhyss Journal, $n^{\circ}$ 05, Juin 2006, pp 75-89.

REMINI B., HALLOUCHE W. (2007). Studying sediment at Algeria's dams. Int Water Power Dam Construction 2007, Vol. 59, pp 42-45.

SEMCHA A. (2006). Valorisation des sédiments de dragage, Applications dans le BTP, cas du barrage de Fergoug. Thèse de Doctorat de l'Université de Reims ChampagneArdenne, 167 p. 